\title{
Estudio de la fluorellesteadita por difracción de rayos $X$ : Propuesta de nuevas líneas de difracción
}

\author{
F. PUERTAS MAROTO; M. T. BLANCO-VARELA; J. L. SAGRERA-MORENO
}

IETCC (CSIC)

\section{$R E S U M E N$}

Se sintetiza el $\mathrm{Ca}{ }_{10}\left(\mathrm{SiO}_{4}\right)_{3}\left(\mathrm{SO}_{4}\right)_{3} \mathrm{~F}_{2}$ y estudian las reflexiones obtenidas por $D R X$, comparándolas con la registrada en la ficha _.C.P.D.S. 23-113 correspondiente al mismo compuesto.

Se propone un nuevo listado que incluye 27 nuevas reflexiones.

Así mismo se modifican algunos valores de las reflexiones dadas en la ficha 23-113.

\section{$S U M M A R Y$}

The $\mathrm{Ca}{ }_{10}\left(\mathrm{SiO}_{4}\right)_{3}\left(\mathrm{SO}_{4}\right)_{3} \mathrm{~F}_{2}$ is synthetized and the reflexions obtained by DRX are studied, comparing them with this registered in the J.C.P.D.S. file which corresponds to the same component.

A new listing which includes 27 new reflexions is proposed.

Some values of the reflexions given in the file 23-113 are modified as well.

\section{INTRODUCCION}

El uso del $\mathrm{CaF}_{2}$ como mineralizador en el proceso de clinkerización ha sido objeto de numerosos estudios en los últimos años (1) (2). Cuando este mineralizador se utiliza conjuntamente con el $\mathrm{CaSO}_{4}$ se obtiene el compuesto $\mathrm{Ca}_{10}\left(\mathrm{SiO}_{4}\right)_{3}\left(\mathrm{SO}_{4}\right)_{3} \mathrm{~F}_{2}$, denominado fluorellesteadita, como fase intermedia.

$\mathrm{La}$ fluorellesteadita es un compuesto cuaternario del sistema de equilibrio $\mathrm{CaO}-\mathrm{SiO}_{2}-\mathrm{CaF}_{2}-\mathrm{CaSO}_{4}$. Giliolli y otros (3) determinaron las relaciones de equilibrio en estado sólido a $1.000^{\circ} \mathrm{C} \mathrm{y}$ $1.150^{\circ} \mathrm{C}$ de este sistema.

Este compuesto funde incongruentemente a $1.250^{\circ} \mathrm{C}(4)$ y cristaliza en el sistema hexagonal, su grupo espacial es el P63/M y los parámetros de celda son: $\mathrm{a}=9,43 \mathrm{y} \mathrm{c}=6,93$ (5).

Sus disoluciones sólidas y sustituciones isomorfas han sido estudiadas por Pliego-Cuervo y otros (6) y Vázquez y otros (7).

En trabajos precedentes (8) y (9) realizados en el Instituto "Eduardo Torroja" se abordó el ahorro de energia en la clinkerización a través de la sustitución parcial de las fases aluminicas y ferriticas, por una fase del tipo fluorellesteadita.

Dada la importancia de este compuesto, como fase intermedia, en el proceso de formación de clínkeres de bajo consumo energético, todos aquellos datos que faciliten su caracterización e identificación tienen un gran interés. 
La fluorellesteadita sintética, así como la obtenida en los trabajos de clinkerización llevados a cabo en nuestros laboratorios presentan algunas diferencias en las líneas de difracción de rayos $\mathrm{X}$ respecto a las propuestas por Kreidler y Hummel (5) que están recogidas en la ficha 23-113 del J.C.P.D.S.

El objeto de este trabajo es aportar nuevos datos que permitan una mejor identificación de la fluorellesteadita por difracción de rayos $\mathrm{X}$.

\section{TRABAJO EXPERIMENTAL}

La muestra se preparó utilizando los reactivos puros de análisis $\mathrm{CaCO}_{3}, \mathrm{CaSO}_{4} \cdot 2 \mathrm{H}_{2} \mathrm{O}, \mathrm{CaF}_{2}$ y $\mathrm{SiO}_{2}$ (Kieselgel) debidamente dosificados y molidos en un molino de ágata.

La muestra introducida en crisol de platino fue sometida a dos periodos de calentamiento de dos horas de duración cada uno, a la temperatura de $1.000 \pm 10^{\circ} \mathrm{C}$; previo al segundo calentamiento se efectuó una molienda.

El compuesto obtenido se estudió por difracción de rayos $\mathrm{X}$, utilizando un equipo automático marca Philips, tipo PW-1700.

Las condiciones de trabajo fueron:

- ánodo de cobre,

$-\mathrm{Kv}=40$,

$-\mathrm{mA}=50$,

- step size $=0,01$,

- count time $=2$ segundos,

- step scan,

- rango angular de $2 \theta=5^{\circ}$ a $2 \theta=75^{\circ}$.

Se realizaron tres registros diferentes de la muestra estudiada con una reproductividad en los valores de $2 \theta$ de $\pm 0,02$ unidades de grado.

Se procedió, posteriormente, a la identificación de la fase, eliminación de las reflexiones correspondientes a las impurezas detectadas y analizar cristalográficamente las reflexiones pertenecientes a la fluorellesteadita.

Como comprobación de resultados, la fluorellesteadita fue sintetizada en iguales condiciones a las descritas por Kreidler y Hummel; es decir utilizando como reactivos $\mathrm{Ca}_{2} \mathrm{SiO}_{4}, \mathrm{Ca}_{2} \mathrm{~F}$ y $\mathrm{CaSO}_{4} \cdot 2 \mathrm{H}_{2} \mathrm{O}$, que dosificados y molidos, fueron calentados a $1.000^{\circ} \mathrm{C}$ durante dos horas. La muestra obtenida fue estudiada por difracción de rayos $\mathrm{X}$, en iguales condiciones a las descritas anteriormente.

\section{RESULTADOS Y DISCUSION}

Para cada registro efectuado a la muestra se obtuvo un listado de las reflexiones $\mathrm{K}_{\alpha 1}$. A estas reflexiones se les asignó sus correspondientes valores de hkl, para una red hexagonal, con grupo espacial $\mathrm{P} 63 / \mathrm{M}$ y parámetros $\mathrm{a}=9,43 \mathrm{y} \mathrm{c}=6,93(5)$. 
TABLA 1

\begin{tabular}{|c|c|c|c|c|c|}
\hline \multicolumn{3}{|c|}{ AUTORES } & \multicolumn{3}{|c|}{ K y H } \\
\hline d & $\mathbf{I} / \mathbf{I}_{0}$ & h K 1 & d & $\mathbf{I} / \mathbf{I}_{0}$ & h K 1 \\
\hline 8.08 & 3 & 100 & & & \\
\hline 5.25 & 1 & 101 & & & \\
\hline 4.69 & 1 & 110 & & & \\
\hline 4.06 & 4 & 200 & 4.03 & 6 & 200 \\
\hline 3.88 & 5 & 111 & 3.92 & 10 & 111 \\
\hline 3.50 & 1 & 201 & 3.53 & 10 & 201 \\
\hline 3.45 & 27 & 002 & 3.47 & 35 & 002 \\
\hline 3.18 & 10 & 102 & 3.20 & 16 & 102 \\
\hline 3.07 & 14 & 210 & 3.09 & 20 & 210 \\
\hline 2.81 & 100 & 211 & 2.82 & 100 & 211 \\
\hline 2.78 & 40 & 112 & 2.80 & 55 & 112 \\
\hline 2.71 & 57 & 300 & 2.73 & 50 & 300 \\
\hline 2.63 & 26 & 202 & 2.64 & 30 & 202 \\
\hline 2.53 & 5 & 301 & 2.54 & 4 & 301 \\
\hline 2.30 & 7 & 212 & 2.31 & 6 & 212 \\
\hline 2.26 & 28 & 310 & 2.27 & 20 & 310 \\
\hline 2.23 & 5 & 221 & 2.23 & 6 & 221 \\
\hline 2.15 & 8 & 311 & 2.15 & 4 & 311 \\
\hline 2.13 & 2 & 302 & & & \\
\hline 2.07 & 5 & 113 & 2.08 & 4 & 113 \\
\hline 2.03 & 2 & 400 & & & \\
\hline 2.009 & 5 & 203 & 2.010 & 6 & 203 \\
\hline 1.947 & 32 & 222 & 1.951 & 20 & 222 \\
\hline 1.895 & 17 & 312 & 1.097 & 14 & 312 \\
\hline 1.872 & 4 & 320 & & & \\
\hline 1.848 & 48 & 213 & 1.850 & 30 & 213 \\
\hline 1.807 & 21 & 321 & 1.810 & 14 & 321 \\
\hline 1.781 & 18 & 410 & 1.785 & 10 & 410 \\
\hline 1.758 & 18 & 402 & 1.760 & 14 & 402 \\
\hline 1.732 & 22 & 004 & 1.734 & 16 & 004 \\
\hline 1.648 & 11 & 322 & 1.647 & 10 & 322 \\
\hline 1.632 & 1 & 500 & & & \\
\hline 1.617 & 5 & 313 & & & \\
\hline 1.590 & 3 & 501 & & & \\
\hline 1.571 & 1 & 330 & & & \\
\hline 1.543 & 6 & 420 & & & \\
\hline 1.532 & 6 & 331 & & & \\
\hline 1.510 & 6 & 214 & & & \\
\hline 1.477 & 16 & 502 & & & \\
\hline 1.466 & 2 & 510 & & & \\
\hline 1.455 & 10 & 323 & & & \\
\hline 1.435 & 8 & 511 & & & \\
\hline 1.411 & 5 & 413 & & & \\
\hline 1.376 & 1 & 314 & & & \\
\hline 1.351 & 4 & 512 & & & \\
\hline 1.343 & 1 & 430 & & & \\
\hline 1.330 & 1 & 115 & & & \\
\hline 1.318 & 7 & 431 & & & \\
\hline 1.308 & 4 & 520 & & & \\
\hline 1.299 & 1 & 333 & & & \\
\hline 1.285 & 4 & 521 & & & \\
\hline 1.272 & 2 & 324 & & & \\
\hline
\end{tabular}


Posteriormente, se procedió a un proceso de refinado de los parámetros $\left({ }^{*}\right)$. Los valores de a y c obtenidos en los tres registros fueron:

$$
\begin{array}{ll}
\mathrm{a}=9.43025 & \mathrm{c}=6.92943 \\
\mathrm{a}=9.43047 & \mathrm{c}=6.93027 \\
\mathrm{a}=9.43140 & \mathrm{c}=6.92852
\end{array}
$$

En los tres casos los valores obtenidos son concordantes con los propuestos por Kreidler y Hummel $(\mathrm{a}=9.43 \mathrm{y} \mathrm{c}=6.93)$.

En la muestra preparada según Kreidler y Hummel y sometida al proceso de refinado, los valores de los parámetros obtenidos fueron $\mathrm{a}=9.43180 \mathrm{y} \mathrm{c}=6.93021$, ambos concordantes con los asignados por ellos.

En la tabla 1, aparece el listado propuesto por los autores y el de Kreidler y Hummel, que corresponde a la ficha 23-113 del J.C.P.D.S.

Comparando ambos listados se observa que para un rango desde $d=17,658\left(2 \theta=5^{\circ}\right)$ hasta $\mathrm{d}=1,6681\left(2 \theta=55^{\circ}\right)$ aparecen seis nuevas reflexiones. Giliolli y otros (10) ya habían informado de la existencia de estas reflexiones. En este rango cabe destacar también que para valores altos, los " $\mathrm{d}$ " propuestos en este trabajo se diferencian notablemente de los propuestos por Kreidler y Hummel mientras que al ir disminuyendo " $\mathrm{d}$ " dichos valores son más coincidentes. Comparando los valores de " $\mathrm{d}$ " relacionados en la tabla I con las teorias de una red con el grupo espacial $\mathrm{P} 63 / \mathrm{M}$ y a $=9,43, \mathrm{c}=6,93$ se encuentra, en el rango mencionado, mayor concordancia en los valores de las reflexiones propuestas por los autores que en los propuestos por Kreidler y Hummel.

En el rango de $d=1,6681\left(1 \theta=55^{\circ}\right)$ a $d=1,2654\left(2 \theta=75^{\circ}\right)$, registro no efectuado por Kreidler y Hummel se proponen 21 nuevas reflexiones.

Las 27 nuevas reflexiones discutidas anteriormente, también aparecen en el registro de la muestra sintetizada en las conclusiones descritas por Kreidler y Hummel.

\section{CONCLUSION}

Para una mejor identificación de la fluorellesteadita por difracción de rayos $\mathrm{X}$ se propone un nuevo listado (alternativo al actual del J.C.P.D.S.) que incluye 27 nuevas reflexiones.

\section{B I B L I O G R A F I A}

(1) KLEMM, W. A. y SKALNY, Y.: "Mineralizers and Fluxes in the clinkering”. Cements Research Progress capitulo XIV (1976).

(2) BUCCHI, R.: "Features on the role of minor compounds in cement clinker. Partes 1 y 2". World Cement Technology. Junio pp. 210-231. Julio/Agosto pp. 258-273 (1981).

(3) GILIOLli, C.; MASSAZA, F.; PEZZUOLI, M.: "Studies on klinker Calcium Silicates bearing $\mathrm{CaF}_{2}$ and $\mathrm{CaSO}_{4}$ ". Cement and Concrete Research. Vol. 9 pp. 295-302 (1979).

(4) BLANCO VARELA, M. T.: "Influencia de Mineralizadores $\mathrm{CaF}_{2}$ y $\mathrm{CaSO}_{4}$, en la formación de silicato bicálcico como contribución en ahorro de energia en el proceso de clinkerización del cemento portland". Tesis doctoral Universidad de Valladolid (1980).

(*) El proceso de refinado consiste en obtener unos valores en los parámetros de celda, correspondientes a un determinado grupo espacial, con los cuales las diferencias entre los valores de $2 \theta$ de las reflexiones teóricas y las obtenidas en la muestra sean lo menor posible. 
(5) KREIDLER, E. R. and HUMMEL, F. A.: "The Crystal Chemistry of Apatite: Structurefields of Fluor and Chlorrapatite" The American Mineralogist. Vol. 55, pp. 170-184, Enero-Febrero (1970).

(6) Pliego-CUERVO, Y.; GLASSER, F. P.: "Phase Relations and Crystal Chemistry of Apatite and Silicocarnotite Solid Solutions". Cement and Concrete Research vol. 8, pp. 519-524 (1978).

(7) BLANCO-VARELA, M. T. y VAZQUEZ-MORENO, T.: "Ahorro de energia en la clinkerización empleando $\mathrm{CaF}_{2}$ y $\mathrm{CaSO}_{4}$ como mineralizadores. Estudio de la fluorellesteadita". Materiales de Construcción, n. ${ }^{\circ} 181$, pp. 55-64, (1981).

(8) BlANCO-VAREla, M. T.; VAzQUEZ MORENO, T.; PAlOMO SANCHEZ, A.: "Utilización de cenizas volantes y mineralizadores como materia prima en la fabricación de cemento". Materiales de Construcción, n. ${ }^{\circ} 189$, pp. 45-54, (1983).

(9) BLANCO-VARELA, M. T.; VAZQUEZ MORENO, T.: "Estudio por espectroscopia IR de un nuevo cemento portland" IX Reunión Nacional de Espectroscopia. Congreso Ibérico de Espectroscopia. Salamanca - Coimbra. 2-7 Octubre, (1985).

(10) GILIOLLI, C.; MASSAZA, F.; PEZZUOLI, M.: "A New compound containing five components 17,2 $\mathrm{CaO} .8 \mathrm{~K}_{2} \mathrm{O}$. .6 $\mathrm{SiO}_{2} .3 \mathrm{SO}_{3} \cdot \mathrm{CaF}_{2}$ ”. Cement and Concrete Research vol. 9; pp. 695-699, (1979).

\section{publicaciones del i.e.t.c.c.}

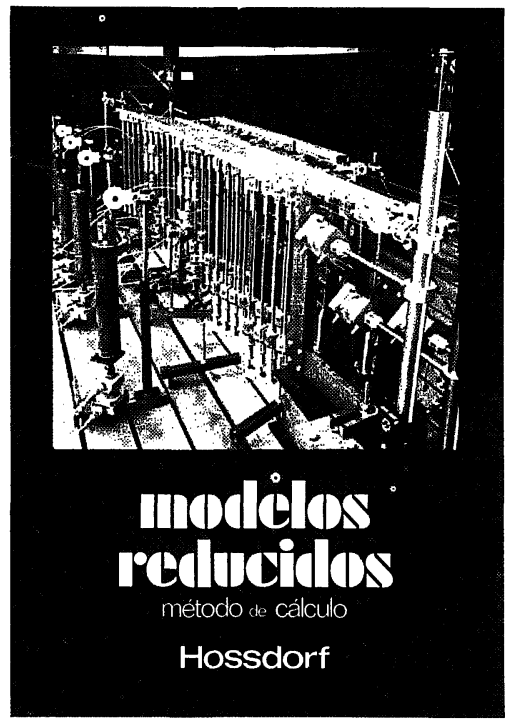

Modelos reducidos. Método de cálculo

H. Hossdorf, Ingeniero Civil

La técnica de los ensayos en modelos reducidos de estructuras sufre hoy dia una decisiva metamorfosis. Hasta hace poco era un medio más bien de artesania, que no siempre era tomado en serio por los académicos teorizan miento resistente de las estructuras complejas y a que se acudio las más de las veces, como a un último remedio debido a sus indiscutibles insuficiencias. Sin embargo, en poco tiempo y gracias a su conexión con los ordenadores digitales, se ha transformado en un instrumento cientificamente valioso que no puede quedar a un lado en la práctica diaria del Ingeniero Proyectista.

Un volumen encuadernado en cartoné plastificado con lomo de tela, de $17 \times 24 \mathrm{~cm}$, compuesto d 250 páginas, 158 figuras y fotografias.

Precios: 1.800 ptas.; \$ USA 26.00

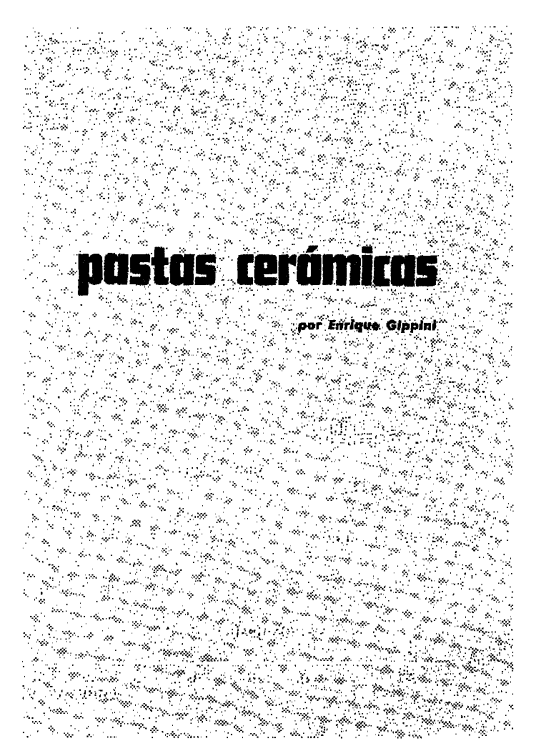

PASTAS CERAMICAS

Enrique Gippini,

Dr. en Ciencias Quimicas

El nexo de unión de todos los capitulos del libro es la idea subyacente de crear una teoria general de pastas. Moldeo y Cocción son los dos procesos a los que debe adecuarse la composición. Las caracteristicas fisico-quimicas más importantes que deben presentar las pastas para que los resultados de estos presentar las pastas para que los resultados de estos proceson satisfactorios $y$ como pueden cambiasescogidos.

Un volumen encuadernado en cartoné, de $25 \times 17 \mathrm{~cm}$ compuesto de 259 páginas, 143 figuras y fotografias, y 37 tablas.

Precios: 2.000 ptas. S USA 29.00

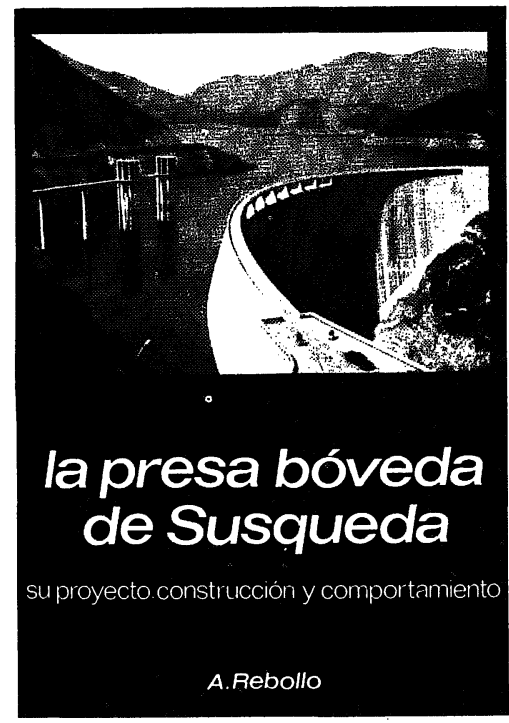

La presa bóveda de Susqueda

A. Rebollo,

Dr. Ingeniero de Caminos

El esfuerzo del constructor de presas se sitúa por su pretensión de perennidad, a contracorriente de las tendencias de la civilización actual, caracterizada por lo fungible. Pueden evocarse las 10.000 grandes presas en funcionamiento o en construcción que están envejeciendo y reclaman los cuidados gerontológicos para mantener y perfeccionar su servicio $y$ car parar su inalienable pretensión perennidad En la medida en que todas nuevas obras, grandes pequeñas, son portadoras de riesgos ecológicos $y$, a veces, catastróficos, que aumentan con el envejecimiento, la gerontologia de las presas es todo un emplazo. La accion adelantada de Arturo Rebollo en este terreno marca un camino a seguir para todos los que aman su propia obra con la devoción paternal que él ha puesto en Susqueda.

Un volumen encuadernado en cartoné plastificado con lomo de tela, de $18 \times 24,5 \mathrm{~cm}$, compuesto de 408 páginas, 330 figuras y fotografias y 39 tablas. Precios: 1.700 ptas.; extranjero, \$ USA 24.00 . 